\title{
Mori Folium water extract alleviates articular cartilage damages and inflammatory responses in monosodium iodoacetate-induced osteoarthritis rats
}

\author{
JIN-WOO JEONG ${ }^{1,2}$, HYE HYEON LEE ${ }^{1,2}$, JONGSIK KIM $^{3}$, EUN-OK CHOI ${ }^{1,2}$, HYUN HWANG-BO $^{1,2}$, \\ HONG JAE KIM ${ }^{1,2}$, MIN YOUNG KIM ${ }^{1,2}$, KYU IM AHN ${ }^{1,2}$, GI-YOUNG KIM ${ }^{4}$, KI WON LEE ${ }^{5}$, KI YOUNG KIM ${ }^{5}$, \\ SUNG GOO KIM ${ }^{5}$, SU HYUN HONG ${ }^{2}$, CHEOL PARK $^{6}$, HEE-JAE CHA ${ }^{7}$ and YUNG HYUN CHOI ${ }^{1,2}$ \\ ${ }^{1}$ Open Laboratory for Muscular and Skeletal Disease and Anti-Aging Research Center, Dongeui University, Busan 614-714; \\ ${ }^{2}$ Department of Biochemistry, Dongeui University College of Korean Medicine, Busan 614-052; \\ ${ }^{3}$ Department of Anatomy, Kosin University College of Medicine, Busan 602-702; ${ }^{4}$ Laboratory of Immunobiology, \\ Department of Marine Life Sciences, Jeju National University, Jeju 690-756; ${ }^{5}$ Research Institute, Bio-Port Korea, Inc., \\ Marine Bio-industry Development Center, Busan 619-912; ${ }^{6}$ Department of Molecular Biology, College of \\ Natural Sciences and Human Ecology, Dongeui University, Busan 614-714; ${ }^{7}$ Department of Parasitology \\ and Genetics, Kosin University College of Medicine, Busan 602-702, Republic of Korea
}

Received March 8, 2017; Accepted June 21, 2017

DOI: $10.3892 / \mathrm{mmr} .2017 .7075$

\begin{abstract}
Mori folium, the leaf of Morus alba L. (Moraceae), has been widely used in traditional medicine for the treatment of various diseases. It has been recently reported that Mori folium possesses potential chondroprotective effects in interleukin (IL)-1 $\beta$-stimulated human chondrocytes; however, its protective and therapeutic potential against osteoarthritis (OA) in an animal model remains unclear. In this study, as part of an ongoing screening program to evaluate the anti-osteoarthritic potential of Mori folium, the protective effects of a water extract of Mori folium (MF) on cartilage degradation and inflammatory responses in a monosodium iodoacetate (MIA)-induced OA rat model were evaluated. The results demonstrated that administration of MF had a tendency to attenuate the damage to articular cartilage induced by MIA, as determined by knee joint swelling and the histological grade of OA. The elevated levels of matrix metalloproteinases-13 and two bio-markers for the diagnosis and progression of OA, such as the cartilage oligomeric matrix protein and $\mathrm{C}$-telopeptide of
\end{abstract}

Correspondence to: Dr Hee-Jae Cha, Department of Parasitology and Genetics, Kosin University College of Medicine, Gamcheollo 262, Seo-gu, Busan 602-072, Republic of Korea

E-mail: hcha@kosin.ac.kr

Dr Yung Hyun Choi, Department of Biochemistry, Dongeui University College of Korean Medicine, Yangjeonglo 52-57, Busan 614-052, Republic of Korea

E-mail: choiyh@deu.ac.kr

Key words: Mori folium, osteoarthritis, monosodium iodoacetate, cartilage degradation, inflammatory responses type II collagen, were markedly ameliorated by MF administration in MIA-induced OA rats. In addition, MF significantly suppressed the production of pro-inflammatory cytokines, including IL-1 $\beta$, IL- 6 and tumor necrosis factor- $\alpha$. MF also effectively inhibited the expression of inducible nitric oxide (NO) synthase and cyclooxygenase-2, thus inhibiting the release of $\mathrm{NO}$ and prostaglandin $\mathrm{E}_{2}$. Although further work is required to fully understand the critical role and clinical usefulness, these findings indicate that MF may be a potential therapeutic option for the treatment of OA.

\section{Introduction}

Human osteoarthritis (OA) is the most frequently occurring degenerative joint disease, causing chronic pain and joint disability worldwide. Although various factors cause OA, aging is the most common factor associated with the development of $\mathrm{OA}$; other factors, such as mechanical stress and hereditary and environmental factors, also lead to the development of OA $(1,2)$. OA is primarily characterized by joint swelling due to a progressive breakdown and loss of articular cartilage, involving increased synovial inflammation, subchondral bone sclerosis and osteophyte formation, which leads to chronic pain and functional limitations in the joint $(3,4)$. Furthermore, the dysregulation of glucose and energy metabolism also aggravate the symptoms in patients with OA (5). In particular, the progressive degradation of the articular cartilage in $\mathrm{OA}$ is mediated by the concerted activation of catabolic tissue proteinases, including matrix metalloproteinases (MMPs), collagenases and aggrecanases, which are upregulated by oxidative stress and other inflammatory stimuli, including pro-inflammatory cytokines and their mediators (6-8). Currently, pharmacological treatment for patients with OA is based on certain steroidal and non-steroidal anti-inflammatory 
drugs (NSAIDs) for the alleviation of pain and inflammation. However, they cannot fully prevent progressive cartilage degradation or repair the impaired cartilage of patients with $\mathrm{OA}$, and long-term use of these drugs can lead to toxicity or severe side effects, including gastrointestinal disturbances and cardiovascular dysfunction (6,9). Therefore, an agent that inhibits cartilage degradation with improved safety and efficacy is an attractive strategy for treatment of OA.

Several researchers have investigated traditional herbal medicinal resources as adjuvant therapeutic agents in the treatment of OA $(10,11)$. Mori folium is the leaf of Morus alba $\mathrm{L}$. (Moraceae) and belongs to the Moraceae family; it has been widely used in traditional medicine from ancient times to the present for the treatment of various diseases in Asian countries, including Korea, China and Japan (12). Mori folium has diverse pharmacological uses, including as an antioxidant (13). It is also known for its antitumor (14), antihypotensive (15), anti-inflammatory (16) and antidiabetic properties (17). A recent study demonstrated that a water extract of Mori folium (MF) possesses potential chondroprotective effects against the collagen matrix breakdown in a pro-inflammatory cytokine interleukin (IL)-1 $\beta$-induced model in vitro (18). However, the in vivo therapeutic effects against $\mathrm{OA}$ and molecular mechanisms of MF have remained elusive. Thus, the present study evaluated the anti-osteoarthritic potential of MF in a rat model. A metabolic inhibitor, monosodium iodoacetate (MIA), was used to induce a form of OA that mimics the structural and biochemical changes associated with human OA (19).

\section{Materials and methods}

Preparation of $M F$. The dried leaves of Morus alba $\mathrm{L}$. were obtained from Bio Port Korea, Inc. (Busan, Korea) and was prepared as previously described (18). In brief, the powdered air-dried leaves were boiled with distilled water $(50 \mathrm{~g} / 500 \mathrm{ml})$ for $3 \mathrm{~h}$. The extracts were filtered through Whatman no. 3 filter paper (Sigma-Aldrich; Merck KGaA, Darmstadt, Germany) twice to remove any insoluble materials, and the filtrate was lyophilized and then crushed into a thin powder. The MF extract was then dissolved to a $100 \mathrm{mg} / \mathrm{ml}$ concentration with distilled water as a stock solution and then diluted with a physiological saline solution to the desired concentration prior to use. A voucher specimen was deposited in the Department of Biochemistry, Dongeui University College of Korean Medicine (Busan, Korea).

Animals. Male Sprague Dawley rats weighing 180-240 g ( 5 weeks old; $n=40$ ) at the start of the study were obtained from Samtako Bio, Inc. (Osan, Korea). All animals were housed two per poly-carbonate cage in a room with controlled temperature conditions $\left(20-24^{\circ} \mathrm{C}\right.$, humidity $\left.40-70 \%\right)$ and controlled lighting ( $12 \mathrm{~h}$ light and/or $12 \mathrm{~h}$ dark cycle). The animals had access to sterile food and water (20). All animal procedures were performed in strict accordance with the recommendations in the Guide for the Care and Use of Laboratory Animals of the National Institutes of Health (21). In addition, the animal protocol used in this study was reviewed and approved by Dongeui University's Institutional Animal Care and Use Committee for compliance with their ethical procedures and scientific care parameters (permit no. A2015-019).

Development of OA with MIA injections and MF administration. The rats were randomly divided into five groups, and each group comprising of 8 rats. For induction of OA, the right knee of the rats were shaved and disinfected with $70 \%$ alcohol following anaesthetization using isoflurane (Baxter International, Inc., Deerfield, IL, USA). On day 0, for all groups of rats, excluding the control and MF only groups, a single injection of $50 \mu \mathrm{l}$ sterile $0.9 \%$ saline containing $3 \mathrm{mg} / \mathrm{kg}$ MIA (Sigma-Aldrich; Merck KGaA) was administered into the right knee joint by the patellar ligament using a $0.3 \mathrm{ml}$ insulin syringe (BD Biosciences, Franklin Lakes, NJ, USA) fitted with a 29-G needle. Control and MF only rats were injected with an equivalent volume of saline. MF was administered orally once per day for 3 weeks at a dose of $100 \mathrm{mg} / \mathrm{kg}$. The rats injected with $3 \mathrm{mg} / \mathrm{kg}$ of celecoxib (LKT Laboratories, Inc., St. Paul, MN, USA) were used as a positive control. All the rats were sacrificed by cervical dislocation, and serum and articular cartilage were collected for subsequent analysis.

Measurement of knee joint swelling. The rats were sacrificed at 3 weeks post-MIA injection, the right knee was isolated, and the femur, tibia, and patella were dissected free of muscle. The knee diameter was measured using a calibrated digital caliper (Mitutoyo Corporation, Kawasaki, Japan) to assess the developmental OA stages 3 weeks post-MIA injection (22).

Joint histological examination. Histological changes were assessed to confirm the effects of MF and celecoxib on cartilage degeneration in the knee joints of MIA-induced $\mathrm{OA}$ rats. Following the rat sacrifice at 3 weeks, each knee joint was resected, fixed in 10\% formalin (Sigma-Aldrich; Merck $\mathrm{KGaA}$ ) for $24 \mathrm{~h}$ at $4{ }^{\circ} \mathrm{C}$, and decalcified with $5 \%$ hydrochloric acid (Sigma-Aldrich; Merck KGaA) for 4 days at $4^{\circ} \mathrm{C}$. Following decalcification specimens were dehydrated in graded acetone and embedded in paraffin. Sections (thickness, $2-3 \mu \mathrm{m}$ ) were stained with $0.2 \%$ hematoxylin and $1 \%$ eosin (H\&E; Sigma-Aldrich; Merck KGaA) for 5 min and $3 \mathrm{~min}$, respectively. For toluidine blue staining, sections were stained with $0.04 \%$ toluidine blue (Sigma-Aldrich; Merck $\mathrm{KGaA}$ ) for 5-10 min, then rinsed in tap water and dried in warm air. For Safranin O (Sigma-Aldrich; Merck KGaA) staining, sections were stained with Weigert's hematoxylin solution for $10 \mathrm{~min}$, and rinsed in running tap water. Then they were placed in fast green solution for $5 \mathrm{~min}$, quickly rinsed in $1 \%$ acetic acid, stained with $0.1 \%$ Safranin O solution for $5 \mathrm{~min}$, and dehydrated in $100 \%$ alcohol at room temperature. Sections were then observed under a Zeiss AxioPlan 2 microscope (Carl Zeiss AG, Oberkochen, Germany). All stained slides were histologically evaluated and statistically graded on a $0-13$ scale by a double-blind observer, according to the modified Mankin scoring system (23).

Immunohistochemical analyses. The sections were depleted of endogenous peroxidase activity by a treatment with $3 \%$ $\mathrm{H}_{2} \mathrm{O}_{2}$ for $15 \mathrm{~min}$ at room temperature. They were then blocked with $10 \%$ normal goat serum (Sigma-Aldrich; Merck KGaA) for $30 \mathrm{~min}$ at room temperature and incubated at $4^{\circ} \mathrm{C}$ overnight 


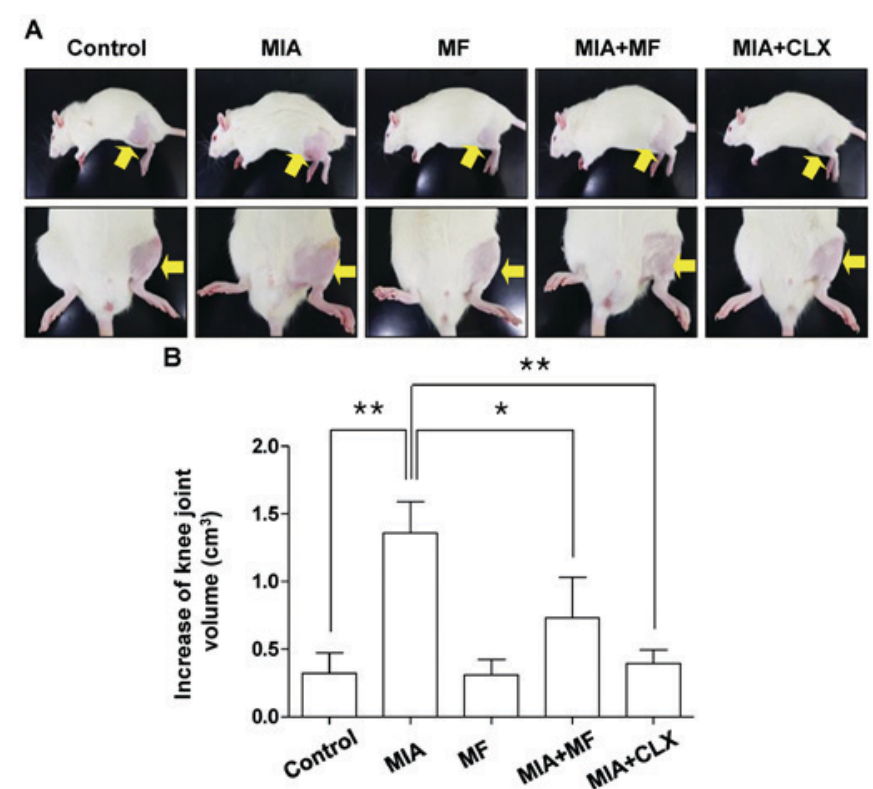

Figure 1. Effects of MF on knee joint swelling in MIA-induced OA rats. The rats were injected with $3 \mathrm{mg}$ MIA in the right knee. Thereafter, MF and CLX were administered orally on a daily basis for 3 weeks after MIA injection (A) Images of the hind knee joint at 3 weeks after the model of MIA-induced OA rats. (B) The severity of OA during the course of MIA was determined by measuring the volume of the hind knee joint using a calibrated digital caliper. Data were expressed as the mean \pm standard deviation $(\mathrm{n}=8)$. ${ }^{*} \mathrm{P}<0.05$ ${ }^{* *} \mathrm{P}<0.01$. MIA, monosodium iodoacetate; MF, Mori folium; CLX, celecoxib.

with primary antibodies, followed by the appropriate biotinylated secondary antibodies (dilution, 1:200) for $30 \mathrm{~min}$ at room temperature and horseradish peroxidase-conjugated streptavidin-biotin staining (cat no. PK-6101; dilution, 1:200; Vector Laboratories, Inc., Burlingame, CA, USA) for $60 \mathrm{~min}$ at room temperature, and finally with $0.1 \%$ 3,3'-diaminobenzidine (dilution, 1:100; Dako; Agilent Technologies, Inc., Santa Clara, CA, USA) for $5 \mathrm{~min}$ at room temperature. The primary antibodies against the following proteins were used: MMP-13 (cat no. ab39012; dilution, 1:50; Abcam, Cambridge, UK), cartilage oligomeric matrix protein (COMP; cat no. ab74524; dilution, 1:50; Abcam), C-telopeptide of type II collagen (CTX-II; cat no. PAA686Hu01; dilution, 1:50; Cloud-Clone Corp., Houston, TX, USA), inducible nitric oxide (NO) synthase (iNOS; cat no. sc-651; dilution 1:100; Santa Cruz Biotechnology, Inc., Dallas, TX, USA), and cyclooxygenase-2 (COX-2; cat no. sc-514489; dilution 1:100; Santa Cruz Biotechnology, Inc.). Histological changes were examined and images by a Carl Zeiss Axio-plan 2 imaging microscope (Carl Zeiss AG).

Serum analysis. At the end of the 3 weeks, the samples of whole blood were collected from the abdominal vein. Blood was allowed to clot for $30 \mathrm{~min}$. Then, the serum was separated via centrifugation at $1,500 \mathrm{x}$ g for $10 \mathrm{~min}$ and stored at $-80^{\circ} \mathrm{C}$ until use. Concentrations of NO in the serum samples were determined by measuring nitrite, which is a major stable product of NO, using the Griess reagent. Briefly, $50 \mu \mathrm{l}$ serum was mixed with $50 \mu \mathrm{l}$ Griess reagent (Sigma-Aldrich; Merck $\mathrm{KGaA}$ ), followed by incubation for $10 \mathrm{~min}$ at $37^{\circ} \mathrm{C}$. Optical density was measured at $540 \mathrm{~nm}$ using an enzyme-linked immunosorbent assay (ELISA) reader (VersaMax; Molecular Devices, LLC, Sunnyvale, CA, USA) (24). The serum levels of cartilage degeneration mediators, such as COMP (cat no. SEB197Ra; Cloud-Clone Corp.) and CTX-II (cat no. MBS700687; MyBioSource, Inc., San Diego, CA, USA), and pro-inflammatory cytokines, including IL-1 $\beta$ (cat no. SRLB00; R\&D Systems, Inc., Minneapolis, MN, USA), IL-6 (cat no. SR6000B; R\&D Systems, Inc.), tumor necrosis factor- $\alpha$ (TNF- $\alpha$; cat no. SRTA00; R\&D Systems, Inc.), and interferon- $\gamma$ (IFN $\gamma$; cat no. SRIF00; R\&D Systems, Inc.) were determined using ELISA kits (R\&D Systems, Inc., Minneapolis, MN, USA), according to the manufacturer's recommendations (25). The level of prostaglandin $\mathrm{E}_{2}\left(\mathrm{PGE}_{2}\right)$ was also determined using an ELISA kit purchased from R\&D Systems, according to the manufacturer's instructions (26).

Statistical analysis. Data were expressed as mean \pm standard deviation for at least three separate determinations for each group. The statistical significance of the differences between groups was assessed using Student's t-test for pair-wise comparisons or a one-way analysis of variance (ANOVA) followed by a Tukey post hoc test for multiple comparisons. Rotarod analysis results were analyzed using a repeated-measures one-way ANOVA followed by a Tukey post hoc test. Statistical analysis was performed using GraphPad Prism software version 5.0 (GraphPad Software, Inc., La Jolla, CA, USA). $\mathrm{P}<0.05$ was considered to indicate a statistically significant difference.

\section{Results}

$M F$ reduces the MIA-induced knee swelling in rats. Knee diameters were measured to determine the degree of joint swelling which is determined by an index of inflammation. As shown in Fig. 1, the knee diameter of the MIA-injected group was increased significantly compared with the control group, and MF alone had little effect. However, MF-administered groups exhibited a significant reduction in knee swelling at 3 weeks after MIA injection. In the celecoxib-treated positive controls, the knee swelling was also decreased to levels near the normal control.

MF ameliorates articular damage in MIA-induced OA rats. As cartilage degeneration is the main histological feature of OA, the effects of MF on the morphological changes and severity of the articular damage were evaluated using H\&E, Safranin O fast green, and toluidine blue staining in the MIA-induced OA rats. The findings demonstrated that the MIA-injected group exhibited severe surface irregularity and surface cleft, and matrix loss of the associated articular cartilage (Fig. 2A). However, administration with MF and celecoxib effectively attenuated the structural morphological changes in the articular cartilages compared with those of the MIA-treated group. Furthermore, the severity of OA lesions was graded using the modified Mankin scoring system, and the overall modified Mankin's scores were significantly reduced in the MF and celecoxib treatment group compared with the MIA only-treated group (Fig. 2B).

MF downregulates MMP-13 expression in MIA-induced OA rats. The progressive destruction associated with cartilage degradation in OA is caused by several matrix-degrading enzymes, including MMPs (27). Among the MMPs family, 

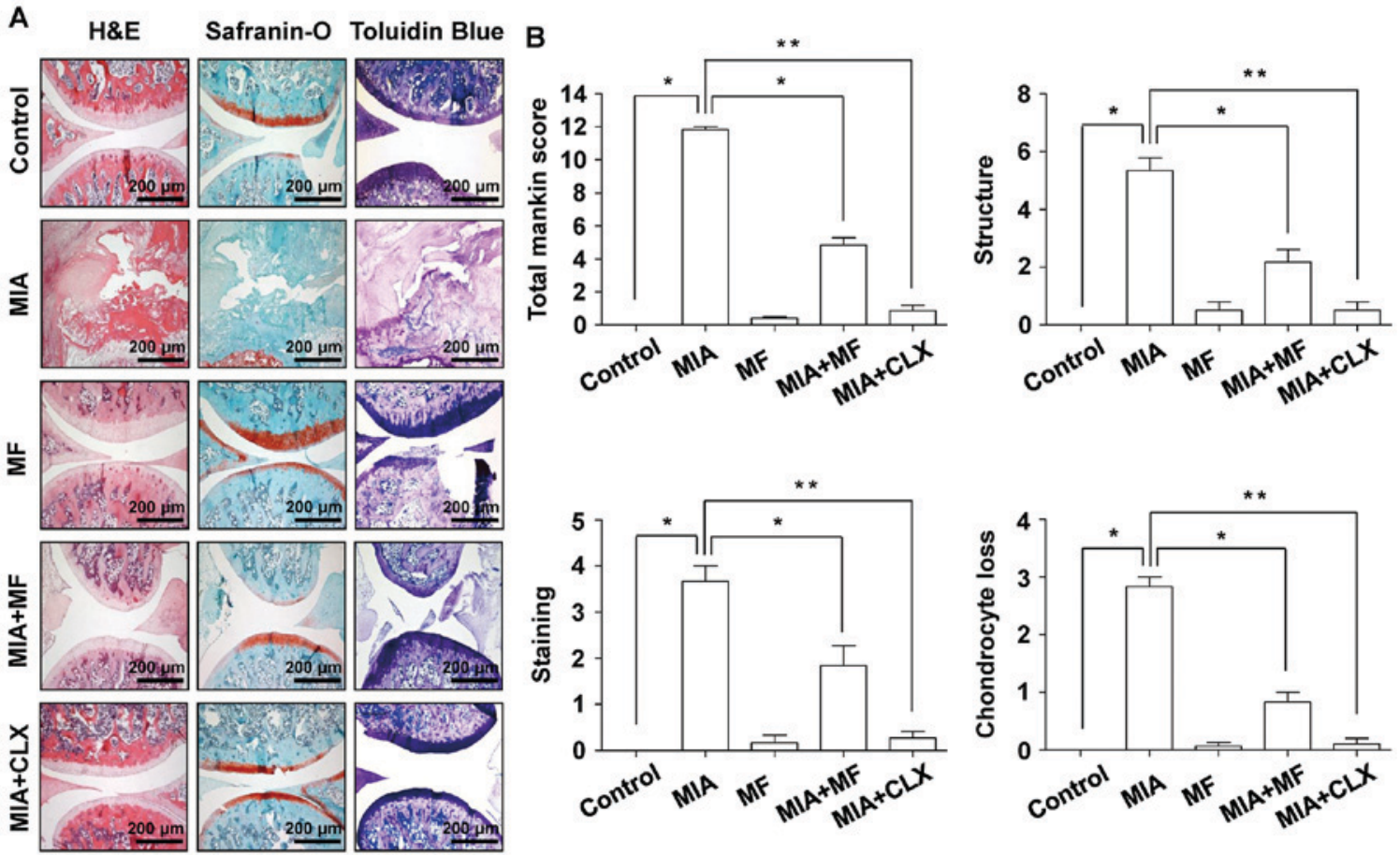

Figure 2. Histological evaluation of joints activity after administration with MF in MIA-induced OA rats. The rats were injected with 3 mg MIA in the right knee, and then CLX was administered orally once a day for 3 weeks after the MIA injection. (A) Knee joints of the OA rats were stained with $\mathrm{H} \& \mathrm{E}$, Safranin-O and toluidine blue. (B) The joint lesions were graded on a scale of 0-13 using the modified Mankin scoring system, giving a combined score for cartilage structure, cellular abnormalities and matrix staining. Data are expressed as the mean \pm standard deviation $(\mathrm{n}=8) .{ }^{*} \mathrm{P}<0.05,{ }^{* *} \mathrm{P}<0.01$. H\&E, hematoxylin and eosin; MIA, monosodium iodoacetate; MF, Mori folium; CLX, celecoxib.

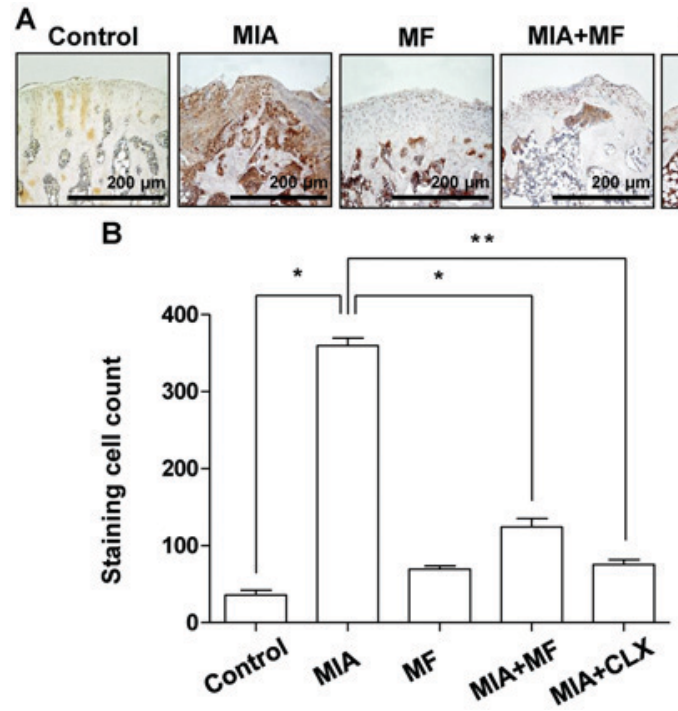

Figure 3. Effects of MF on the expression of MMP-13 in MIA-induced OA rats. (A) Immunohistochemical staining was used to identify the expression of MMP-13 in the articular cartilage. (B) Stained cells wren counted and data are expressed as mean \pm standard deviation $(\mathrm{n}=8)$. ${ }^{*} \mathrm{P}<0.05,{ }^{* *} \mathrm{P}<0.01$. MIA, monosodium iodoacetate; MF, Mori folium; CLX, celecoxib; MMP, matrix metalloproteinase.

MMP-13 has the most important role in degrading cartilage (28); thus, the effects of MF on the expression of MMP-13 were examined in MIA-stimulated OA cartilage. The results demonstrated that the number of chondrocytes staining positive for MMP-13 increased following MIA injection, with significantly lower percentages of MMP-13-positive
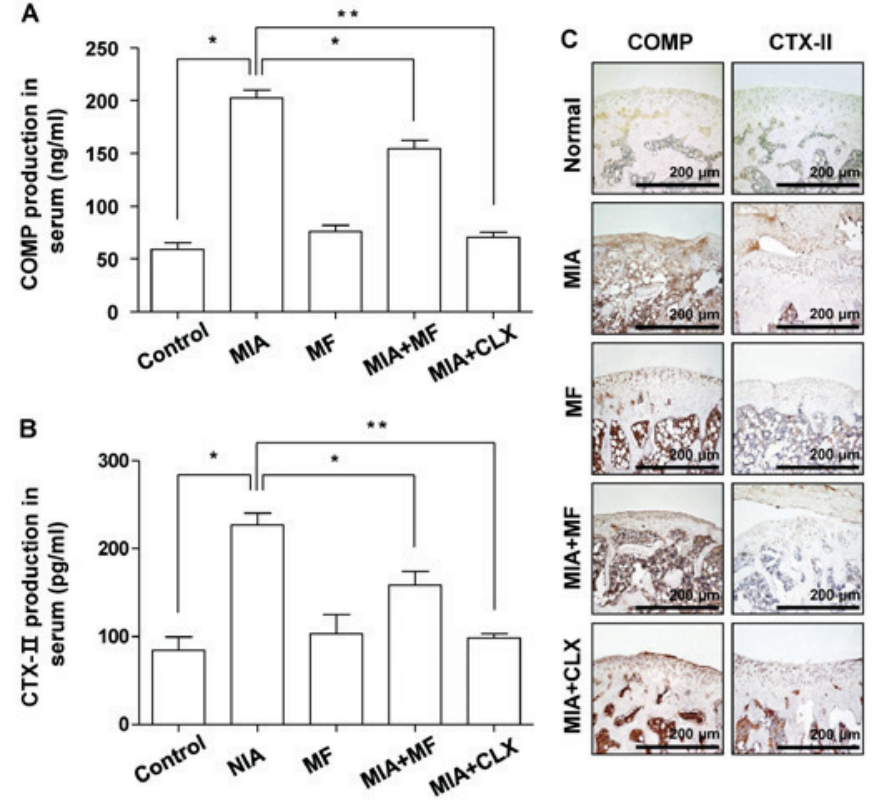

Figure 4. Effects of MF on the production and expression of COMP and CTX-II in MIA-induced OA rats. (A) COMP and (B) CTX-II production was measured in the serum of MIA-induced OA rats using commercial ELISA kits. (C) Immunohistochemical staining was used to identify the expression of COMP and CTX-II in the articular cartilage. Data are expressed as the mean \pm standard deviation $(\mathrm{n}=8)$. ${ }^{*} \mathrm{P}<0.05,{ }^{* *} \mathrm{P}<0.01$. MIA, monosodium iodoacetate; MF, Mori folium; CLX, celecoxib; COMP, cartilage oligomeric matrix protein; CTX-II, C-telopeptide of type II collagen.

chondrocytes observed in the MF- and celecoxib-administered groups compared with the MIA only-treated controls (Fig. 3). 

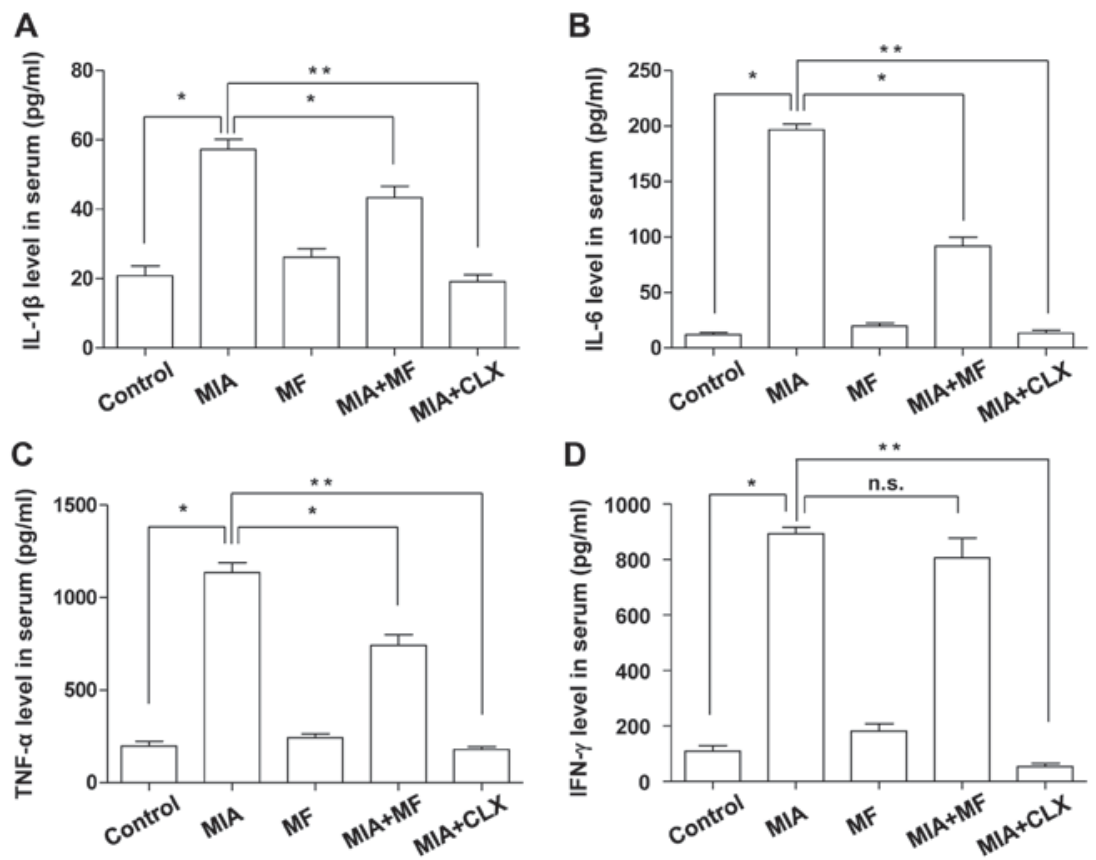

Figure 5. Effects of MF on the level of IL-1 $\beta$, IL-6, TNF- $\alpha$ and IFN- $\gamma$ in the serum of MIA-induced OA rats. The concentrations of (A) IL-1 $\beta$, (B) IL-6, (C) TNF- $\alpha$ and (D) IFN- $\gamma$ in serum collected from MIA-induced OA rats treated with or without MF or CLX were measured. Data are expressed as the mean \pm standard deviation $(\mathrm{n}=8) .{ }^{*} \mathrm{P}<0.05,{ }^{* *} \mathrm{P}<0.01$. IL, interleukin; MIA, monosodium iodoacetate; MF, Mori folium; CLX, celecoxib; TNF- $\alpha$, tumor necrosis factor- $\alpha$; IFN- $\gamma$, interferon- $\gamma ;$ n.s., no significance.
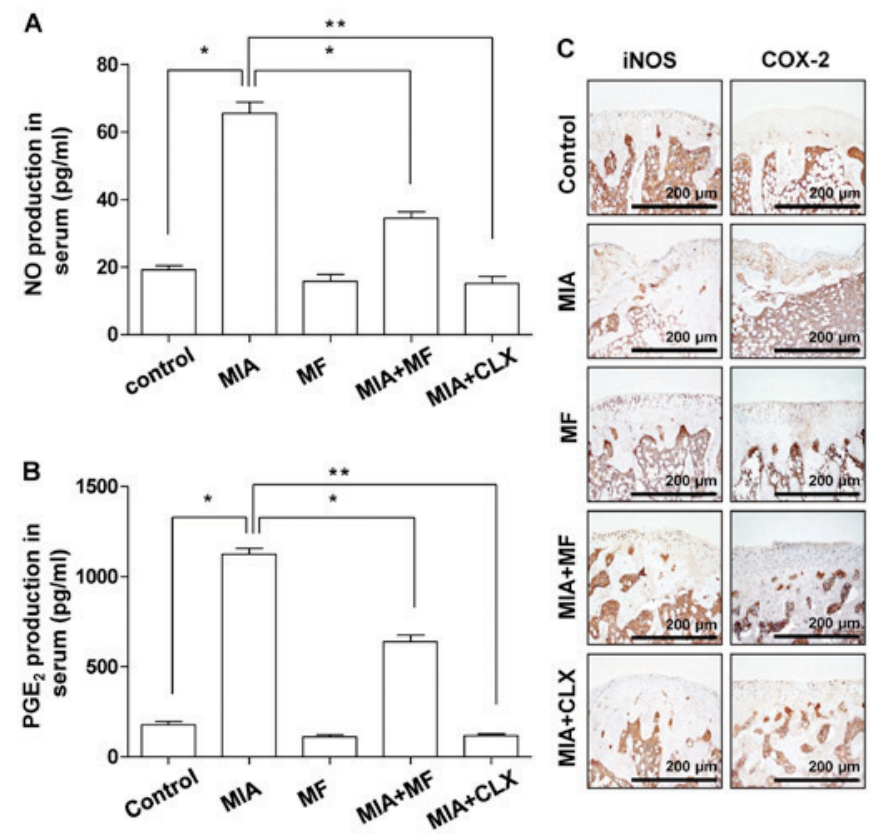

Figure 6. Effects of MF on production of $\mathrm{NO}$ and $\mathrm{PGE}_{2}$, and the expression of iNOS and COX-2 in MIA-induced OA rats. (A) NO and (B) $\mathrm{PGE}_{2}$ contents were measured in the serum of MIA-induced OA rats using Griess reagent and commercial ELISA kits, respectively. Data are the mean \pm standard deviation $(\mathrm{n}=8)$. ${ }^{*} \mathrm{P}<0.05,{ }^{* *} \mathrm{P}<0.01$. (C) Immunohistochemical staining was used to identify the expression of iNOS and COX-2 in the articular cartilage. NO, nitric oxide; MIA, monosodium iodoacetate; MF, Mori folium; CLX, celecoxib; $\mathrm{PGE}_{2}$, prostaglandin $\mathrm{E}_{2}$; iNOS, inducible nitric oxide synthase; COX-2, cyclooxygenase-2.

$M F$ reduces the production and expression of $C O M P$ and CTX-II in MIA-induced OA rats. The effect of MF on the levels of COMP and CTX-II, which are well-established bio-markers for diagnosis and OA progression that have key roles during subsequent progressive osteophytosis in multiple knee compartments $(29,30)$, was also investigated. The ELISA data indicated that MF alone did not significantly affect COMP and CTX-II production at basal levels. However, significantly increased production of COMP and CTX-II were detected in the serum of MIA-induced OA rats; these levels were significantly diminished following $\mathrm{MF}$ administration (Fig. 4A and B). The MIA-induced overproduction of COMP and CTX-II was also blocked by celecoxib. Consistent with the ELISA results, immunocytochemical analysis demonstrated that oral administration of MF and celecoxib caused a significant decrease in the expression of COMP and CTX-II compared with that in the normal control group (Fig. 4C).

$M F$ inhibits the production of pro-inflammatory cytokines in $M I A$-induced $O A$ rats. It was also determined whether MF had any inhibitory effects on the production of inflammatory cytokines associated with OA, including IL-1 $\beta$, IL-6, TNF- $\alpha$ and IFN- $\gamma$, which have important roles in the development and progression of OA $(31,32)$. As presented in Fig. 5, the serum levels of these cytokines remained at a low level in the control group. Compared with the controls, the concentrations of IL- $1 \beta$, IL- 6 , TNF- $\alpha$, and IFN- $\gamma$ in the MIA group were markedly higher; however, the groups treated with MF or celecoxib had significantly reduced production of IL-1 $\beta$, IL-6 and TNF- $\alpha$ compared with the MIA only group. There was no significant difference in the level of IFN- $\gamma$ between the MIA only and MIA + MF group.

Effect of $M F$ on the production of $N O$ and $P G E_{2}$, and the expression of iNOS and COX-2 in MIA-induced OA rats. The 
overproduction of pro-inflammatory mediators, including $\mathrm{NO}$ and $\mathrm{PGE}_{2}$, also has been associated with the pathophysiology of OA $(33,34)$, thus, the effects of MF on NO and $\mathrm{PGE}_{2}$ release were investigated in MIA-induced OA rats. As indicated in Fig. 6A and $\mathrm{B}$, significant increases in the NO and $\mathrm{PGE}_{2}$ levels were detected in the MIA-induced OA rats compared with the normal control group; however, this effect was significantly decreased by administration of MF and celecoxib. Subsequently, immunohistochemical analysis was performed to determine whether the protective effects on $\mathrm{NO}$ and $\mathrm{PGE}_{2}$ production were associated with the changes in the expression of their synthesis enzymes, iNOS and COX-2, in the knee joints. Compared with the control group, MIA markedly induced the expression of iNOS and COX-2, while MF and celecoxib obviously suppressed the elevated expression levels of iNOS and COX-2 in the cartilaginous tissues of the MIA-induced OA rats (Fig. 6C).

\section{Discussion}

MIA-induced OA in rodents is one of the well-established models to describe the anti-osteoarthritic effects of candidate drugs on the pathology of OA. Upon injection of MIA into joints, the activity of GAPDH is inhibited and then leads to the disruption of glycolysis. This process successfully induces degenerative changes in the articular cartilage by the direct interruption of chondrocyte metabolism and the subsequent induction of chondrocyte death, representative of the changes observed in patients with OA (19). In the current study, it was investigated whether MF exerts an anti-osteoarthritic effect in an MIA-induced rat OA model. The data demonstrated that the oral administration of MF led to a significant decrease in structural changes, including joint space narrowing and cartilage destruction, in MIA-induced OA rats, as did celecoxib, which was associated with reductions in MMP-13, COMP, CTX-II, and pro-inflammatory cytokines and mediators.

Increasing evidence indicates that MMPs are important proteases involved in tissue remodeling, including the turnover, catabolism and degradation of the extracellular matrix (ECM) (27). MMP expression can be upregulated by pro-inflammatory cytokines in a variety of tissues and cell types, including articular chondrocytes $(6,27,28)$. Among the MMPs, MMP-13 is critical for degrading collagens, proteoglycans and other ECM macromolecules in the osteoarthritic pathological process (28). Thus, the expression of MMP-13 was analyzed to assess the effects of MF on the catabolic activity of chondrocytes. Consistent with previous studies $(19,35)$, the serum levels of MMP-13 and the percentages of MMP-13-positive chondrocytes were significantly higher in the MIA-treated group than in the saline-treated control and MF only groups. However, the MIA + MF rats had fewer MMP-13-producing cells than the MIA-treated rats, which was also associated reduced MMP-13 production determined by ELISA. The protective effects of MF were comparable to the positive control drug, celecoxib, which is a COX-2-selective NSAID. These observations support the fact that MF may have an anti-osteoarthritic effect by reducing the production and activation of MMP-13.

Several previous studies have demonstrated that COMP, a pentameric glycoprotein, is one of the essential components of the extracellular matrix of the cartilage $(29,30)$. COMP functions as a regulator in governing the assembly of type II collagen fibers in cartilage; thus, this glycoprotein stabilizes the collagen network in cooperation with other matrix proteins (36). However, its levels during the development of $\mathrm{OA}$ and under inflammatory conditions are obviously increased in serum and synovial fluid and positively correlated with joint damage in knee OA $(30,37)$. In addition, CTX-II is produced by the degradation of type II collagen through the action of proteases with cartilage injury or degeneration and finally excreted in the urine $(36,38)$. CTX-II contents were also elevated in OA patients compared with normal individuals, as the levels are associated with both the prevalence and progression of OA $(38,39)$. These observations indicated that these two factors have the potential to be prognostic bio-markers for monitoring cartilage degradation in patients with OA $(29,30)$. The ELISA results in the current study demonstrated that serum COMP and CTX-II levels in the MIA-induced OA group were significantly higher than the control group; however, treatment with MF significantly prevented this increase. In agreement with the ELISA data, immunohistochemistry analysis demonstrated that rats injected with MIA exhibited increased COMP and CTX-II expression in articular cartilage, and administration with MF or celecoxib significantly reduced their expression in MIA-induced OA rats. Therefore, the reduction in COMP and CTX-II serum levels by MF may represent suppressed MIA-induced degradation of cartilage, as cartilage is a major contributor to circulating COMP and CTX-II levels.

Compelling evidence has demonstrated that pro-inflammatory cytokines are significantly elevated in synovial fluid from patients with OA and have critical roles in the promotion of the catabolic processes in OA, causing cartilage degradation $(31,40)$. High levels of pro-inflammatory cytokines have been detected in synovial fluid from patients with OA and several animal models of cartilage degradation (41). Among these cytokines, IL-1 $\beta$ is highly overexpressed in the cartilage and in the synovial tissue, while the expression of IL-1 receptor antagonist (42), inhibits proliferation, triggers apoptosis of chondrocytes, and blocks the synthesis of ECM structural components by activating MMPs, including MMP-13 $(32,40)$. IL-6 has also been reported to act as one of the major pro-inflammatory cytokines involved in the pathophysiology of OA. IL-6 induces the destruction of joint and cartilage by stimulating the activation of osteoclasts and the differentiation of mesenchymal cells into chondroblasts $(42,43)$. In addition, previous studies have revealed that TNF- $\alpha$ is similar to or synergistic with IL- $1 \beta$ and IL- 6 in the production of matrix-degrading enzymes and in the inhibition of proteoglycan synthesis, resulting in loss of cartilage and bone resorption during the process of OA development $(41,42)$. Studies have demonstrated that anti-inflammatory agents capable of inhibiting the production of those cytokines may have the potential to control or treat OA $(31,32)$. Thus, the anti-inflammatory effects of MF were investigated in the present study by measuring the serum levels of pro-inflammatory cytokines (IL-1 $\beta$, IL- 6 and TNF- $\alpha$ ) in MIA-induced OA rats and observed that MF application could greatly diminish the MIA injection-induced increase of these cytokines. Taken together, the present results indicate that MF has the potential 
to deter inflammatory responses and may subsequently reduce damage of articular cartilage.

In addition to the roles of pro-inflammatory cytokines in OA, pro-inflammatory mediators, such as $\mathrm{NO}$ and $\mathrm{PEG}_{2}$, have an important role in the development of inflammation in OA $(40,44)$. The mediators can induce the cell death of chondrocytes and the loss of cartilage matrix in the pathogenesis of OA, and they are also significantly elevated in cartilage and synovial tissues from patients with OA $(45,46)$. Furthermore, the pro-inflammatory cytokines can stimulate the production of pro-inflammatory mediators through the activation of chondrocytes $(34,40)$. Therefore, the effects of MF on the release of $\mathrm{NO}$ and $\mathrm{PEG}_{2}$ were also analyzed in the MIA-induced OA rat model. The results clearly demonstrated that the serum levels of $\mathrm{NO}$ and $\mathrm{PGE}_{2}$ were significantly higher in the MIA group compared with the control group. Although the suppressive property of MF was weaker than that of the celecoxib, MF significantly reduced the MIA-induced elevation of NO and $\mathrm{PGE}_{2}$ production and suppressed the expression of upstream molecules, iNOS and COX-2, which is consistent with the previous report demonstrating that $\mathrm{MF}$ reduced the production of $\mathrm{NO}$ and $\mathrm{PEG}_{2}$ in IL-1 $\beta$-stimulated human chondrocytes (18).

In conclusion, the present study demonstrated that administration with MF effectively attenuated the severity of articular cartilage destruction in MIA-induced OA of the knee joint in rats. To the best of our knowledge, this is the first report to demonstrate the anti-osteoarthritic effects of MF on an MIA-induced OA model. The anti-osteoarthritic effects of MF were associated with the suppression of MMP-13 and two representative bio-markers for the diagnosis of OA, COMP and CTX-II, in the OA animal model induced by MIA. MF also reduced the production of pro-inflammatory cytokines, such as IL-1 $\beta$, IL-6, and TNF- $\alpha$, and mediators (including NO and $\mathrm{PGE}_{2}$ ). Although the results suggest that MF may be a promising compound for treating $\mathrm{OA}$, further studies are required to ascertain the molecular mechanisms of action, and clinical trials to estimate the efficacy of MF in patients with OA.

\section{Acknowledgements}

This work was supported by the High Value-added Food Technology Development Program (grant no. 314043-3), Ministry of Agriculture, Food and Rural Affairs, Republic of Korea.

\section{References}

1. Andriacchi TP, Favre J, Erhart-Hledik JC and Chu CR: A systems view of risk factors for knee osteoarthritis reveals insights into the pathogenesis of the disease. Ann Biomed Eng 43: 376-387, 2015.

2. Wang F, Cai F, Shi R, Wang XH and Wu XT: Aging and age related stresses: A senescence mechanism of intervertebral disc degeneration. Osteoarthritis Cartilage 24: 398-408, 2016.

3. Speziali A, Delcogliano M, Tei M, Placella G, Chillemi M, Tiribuzi R and Cerulli G: Chondropenia: Current concept review. Musculoskelet Surg 99: 189-200, 2015.

4. Guilak F: Biomechanical factors in osteoarthritis. Best Pract Res Clin Rheumatol 25: 815-823, 2011.

5. King KB and Rosenthal AK: The adverse effects of diabetes on osteoarthritis: Update on clinical evidence and molecular mechanisms. Osteoarthritis Cartilage 23: 841-850, 2015.

6. Goldring MB and Berenbaum F: Emerging targets in osteoarthritis therapy. Curr Opin Pharmacol 22: 51-63, 2015.
7. Gargiulo S, Gamba P, Poli G and Leonarduzzi G: Metalloproteinases and metalloproteinase inhibitors in age-related diseases. Curr Pharm Des 20: 2993-3018, 2014.

8. Okada Y: Matrix-degrading metalloproteinases and their roles in joint destruction. Mod Rheumatol 10: 121-128, 2000.

9. Mobasheri A: The future of osteoarthritis therapeutics: Targeted pharmacological therapy. Curr Rheumatol Rep 15: 364, 2013

10. Hou PW, Fu PK, Hsu HC and Hsieh CL: Traditional Chinese medicine in patients with osteoarthritis of the knee. J Tradit Complement Med 5: 182-196, 2015.

11. Cameron $\mathrm{M}$ and Chrubasik S: Topical herbal therapies for treating osteoarthritis. Cochrane Database Syst Rev 31: CD010538, 2013.

12. Chan EW, Lye PY and Wong SK: Phytochemistry, pharmacology, and clinical trials of Morus alba. Chin J Nat Med 14: 17-30, 2016.

13. Kim DS, Kang YM, Jin WY, Sung YY, Choi G and Kim HK: Antioxidant activities and polyphenol content of Morus alba leaf extracts collected from varying regions. Biomed Rep 2: 675-680, 2014.

14. Deepa M, Sureshkumar T, Satheeshkumar PK and Priya S: Antioxidant rich Morus alba leaf extract induces apoptosis in human colon and breast cancer cells by the downregulation of nitric oxide produced by inducible nitric oxide synthase. Nutr Cancer 65: 305-310, 2013.

15. Kobayashi Y, Miyazawa M, Kamei A, Abe K and Kojima T: Ameliorative effects of mulberry (Morus alba L.) leaves on hyperlipidemia in rats fed a high-fat diet: Induction of fatty acid oxidation, inhibition of lipogenesis, and suppression of oxidative stress. Biosci Biotechnol Biochem 74: 2385-2395, 2010.

16. Chao WW, Kuo YH, Li WC and Lin BF: The production of nitric oxide and prostaglandin E2 in peritoneal macrophages is inhibited by Andrographis paniculata, Angelica sinensis and Morus alba ethyl acetate fractions. J Ethnopharmacol 122: $68-75,2009$.

17. Naowaboot J, Pannangpetch $\mathrm{P}$, Kukongviriyapan V, Kukongviriyapan U, Nakmareong S and Itharat A: Mulberry leaf extract restores arterial pressure in streptozotocin-induced chronic diabetic rats. Nutr Res 29: 602-608, 2009.

18. Jeong JW, Lee HH, Lee KW, Kim KY, Kim SG, Hong SH, Kim GY, Park C, Kim HK, Choi YW and Choi YH: Mori folium inhibits interleukin- $1 \beta$-induced expression of matrix metalloproteinases and inflammatory mediators by suppressing the activation of NF- $\kappa$ B and $\mathrm{p} 38$ MAPK in SW1353 human chondrocytes. Int J Mol Med 37: 452-460, 2016.

19. Barve RA, Minnerly JC, Weiss DJ, Meyer DM, Aguiar DJ, Sullivan PM, Weinrich SL and Head RD: Transcriptional profiling and pathway analysis of monosodium iodoacetate-induced experimental osteoarthritis in rats: Relevance to human disease. Osteoarthritis Cartilage 15: 1190-1198, 2007.

20. Lee TH, Song HK, Jang JY, Kim DY, Park HK, Choi EA and Han BS: Anti-inflammatory effect of egg white-chalcanthite and purple bamboo salts mixture on arthritis induced by monosodium iodoacetate in Sprague-Dawley rats. Lab Anim Res 32: 91-98, 2016.

21. National Research Council (US): Committee for the Update of the Guide for the Care and Use of Laboratory Animals. Guide for the care and use of laboratory animals. 8th edition. National Academies Press, Washington, 2011

22. Fernihough J, Gentry C, Malcangio M, Fox A, Rediske J, Pellas T, Kidd B, Bevan S and Winter J: Pain related behaviour in two models of osteoarthritis in the rat knee. Pain 112: 83-93, 2004.

23. Mankin HJ, Dorfman H, Lippiello L and Zarins A: Biochemical and metabolic abnormalities in articular cartilage from osteo-arthritic human hips. II. Correlation of morphology with biochemical and metabolic data. J Bone Joint Surg Am 53: 523-537, 1971.

24. Kwon HO, Lee M, Kim OK, Ha Y, Jun W and Lee J: Effect of Hijikia fusiforme extracts on degenerative osteoarthritis in vitro and in vivo models. Nutr Res Pract 10: 265-273, 2016.

25. Kim MS, Kim JE, Yoon YS, Seo JG, Chung MJ and Yum DY: A probiotic preparation alleviates atopic dermatitis-like skin lesions in murine models. Toxicol Res 32: 149-158, 2016.

26. Woo SJ, Lee SM, Lim HS, Hah YS, Jung ID, Park YM, Kim HO, Cheon YH, Jeon MG, Jang KY, et al: Myeloid deletion of SIRT1 suppresses collagen-induced arthritis in mice by modulating dendritic cell maturation. Exp Mol Med 48: e221, 2016.

27. Troeberg L and Nagase H: Proteases involved in cartilage matrix degradation in osteoarthritis. Biochim Biophys Acta 1824: $133-145,2012$. 
28. Takaishi H, Kimura T, Dalal S, Okada Y and D'Armiento J: Joint diseases and matrix metalloproteinases: A role for MMP-13. Curr Pharm Biotechnol 9: 47-54, 2008

29. Goode AP, Marshall SW, Kraus VB, Renner JB, Stürmer T, Carey TS, Irwin DE and Jordan JM: Association between serum and urine biomarkers and lumbar spine individual radiographic features: The Johnston County Osteoarthritis Project. Osteoarthritis Cartilage 20: 1286-1293, 2012

30. Saberi Hosnijeh F, Siebuhr AS, Uitterlinden AG, Oei EH, Hofman A, Karsdal MA, Bierma-Zeinstra SM, Bay-Jensen AC and van Meurs JB: Association between biomarkers of tissue inflammation and progression of osteoarthritis: Evidence from the Rotterdam study cohort. Arthritis Res Ther 18: 81, 2016.

31. Kapoor M, Martel-Pelletier J, Lajeunesse D, Pelletier JP and Fahmi H: Role of proinflammatory cytokines in the pathophysiology of osteoarthritis. Nat Rev Rheumatol 7: 33-42,2011.

32. Mabey T and Honsawek S: Cytokines as biochemical markers for knee osteoarthritis. World J Orthop 6: 95-105, 2015.

33. Abramson SB, Attur M, Amin AR and Clancy R: Nitric oxide and inflammatory mediators in the perpetuation of osteoarthritis. Curr Rheumatol Rep 3: 535-541, 2001.

34. Martel-Pelletier J, Pelletier JP and Fahmi H: Cyclooxygenase-2 and prostaglandins in articular tissues. Semin Arthritis Rheum 33: 155-167, 2003.

35. Andereya S, Streich N, Schmidt-Rohlfing B, Mumme T, Müller-Rath R and Schneider U: Comparison of modern marker proteins in serum and synovial fluid in patients with advanced osteoarthrosis and rheumatoid arthritis. Rheumatol Int 26: 432-438, 2006

36. Christgau S, Garnero P, Fledelius C, Moniz C, Ensig M, Gineyts E, Rosenquist C and Qvist P: Collagen type II C-telopeptide fragments as an index of cartilage degradation. Bone 29: 209-215 2001.

37. Vilím V, Olejárová M, Machácek S, Gatterová J, Kraus VB and Pavelka K: Serum levels of cartilage oligomeric matrix protein (COMP) correlate with radiographic progression of knee osteoarthritis. Osteoarthritis Cartilage 10: 707-713, 2002.
38. Freeston JE, Garnero P, Wakefield RJ, Hensor EM, Conaghan PG and Emery P: Urinary type II collagen C-terminal peptide is associated with synovitis and predicts structural bone loss in very early inflammatory arthritis. Ann Rheum Dis 70: 331-333, 2011.

39. Jansen NW, Roosendaal G, Lundin B, Heijnen L, Mauser-Bunschoten E, Bijlsma JW, Theobald M and Lafeber FP: The combination of the biomarkers urinary C-terminal telopeptide of type II collagen, serum cartilage oligomeric matrix protein and serum chondroitin sulfate 846 reflects cartilage damage in hemophilic arthropathy. Arhtritis Rheum 60: 290-298, 2009.

40. Rahmati M, Mobasheri A and Mozafari M: Inflammatory mediators in osteoarthritis: A critical review of the state-of-the-art, current prospects, and future challenges. Bone 85: 81-90, 2016.

41. Kellesarian SV, Al-Kheraif AA, Vohra F, Ghanem A, Malmstrom H, Romanos GE and Javed F: Cytokine profile in the synovial fluid of patients with temporomandibular joint disorders: A systematic review. Cytokine 77: 98-106, 2016.

42. Jotanovic Z, Mihelic R, Sestan B and Dembic Z: Role of interleukin-1 inhibitors in osteoarthritis: An evidence-based review. Drugs Aging 29: 343-358, 2012.

43. Doss F, Menard J, Hauschild M, Kreutzer HJ, Mittlmeier T, Müller-Steinhardt M and Müller B: Elevated IL-6 levels in the synovial fluid of osteoarthritis patients stem from plasma cells. Scand J Rheumatol 36: 136-139, 2007.

44. Amin AR, Dave M, Attur M and Abramson SB: COX-2, NO and cartilage damage and repair. Curr Rheumatol Rep 2: 447-453, 2000.

45. Notoya K, Jovanovic DV, Reboul P, Martel-Pelletier J, Mineau F and Pelletier JP: The induction of cell death in human osteoarthritis chondrocytes by nitric oxide is related to the production of prostaglandin E2 via the induction of cyclooxygenase-2. J Immunol 165: 3402-3410, 2000.

46. Park JY, Pillinger MH and Abramson SB: Prostaglandin E2 synthesis and secretion: The role of PGE2 synthases. Clin Immunol 119: 229-240, 2006. 